\title{
FOURIER TRANSFORM SPECTROPHOTOMETRY AND ITS APPLICATION TO THE STUDY OF K-GIANTS
}

\author{
S. T. RIDGWAY \\ Kitt Peak National Observatory, Tucson, Ariz. \\ and \\ State University of New York at Stony Brook, U.S.A.
}

\begin{abstract}
A Fourier Transform Spectrometer has been employed to obtain low and medium resolution spectrophotometry in the range 700 to $10000 \mathrm{~cm}^{-1}$. The method is described briefly and typical data are shown. The technique is applied to a study of $15 \mathrm{~K}$ giants, and spectra of 4 and $16 \mathrm{~cm}^{-1}$ resolution in the range 4000 to $6500 \mathrm{~cm}^{-1}$ are discussed briefly. $\mathrm{CN}$ strong stars are found to be $\mathrm{CO}$ strong as well. Five $\mathrm{K}$ giants are found to have a relative ${ }^{13} \mathrm{C}$ enhancement over probable primordial abundances.
\end{abstract}

\section{Introduction}

For the last several years I have been applying Fourier spectroscopy to several planetary and stellar studies. Although high resolution has been obtained in some cases, much of the work has been done at low resolution employing techniques which might reasonably be called Fourier Transform Spectrophotometry. I will describe briefly the technique, then present typical data and discuss a number of results from a K-giant survey.

\section{Fourier Transform Spectrophotometry}

The scanning interferometer is a standard commercial model (Idealab, Inc., Maynard, Mass.). Maximum mirror travel is $1 \mathrm{~cm}$ and operation is in the rapid scanning mode first described by Mertz. A general discussion of the spectrometer system is available (Ridgway, 1972).

For the spectrometer to be useful beyond $3 \mu$, where thermal background emission is severe, it was especially important that the instrument has two extremely well balanced inputs for star + sky and sky. The foreoptics design is shown in Figure 1. Notice that the input optics are symmetric about the beamsplitter plane. Especially valuable for balancing the beams, two variable apertures are located in the prime focal plane. In addition, the $150 \mathrm{~cm}$ McMath solar telescope at Kitt Peak, which was used for this work, has a completely unobscured aperture, significantly reducing the thermal background problems. The two inputs are readily balanced to better than one part in 100, and moving the star from one aperture to the other between scans reduced the residual imbalance to below the detector noise for up to $\frac{1}{2} \mathrm{~h}$ integrations.

The detectors employed were PbS (Santa Barbara Research Corporation, Santa Barbara, California) with an NEP of $3 \times 10^{-14} \mathrm{~W} \mathrm{~Hz}^{-1 / 2}$ in the range 1 to $4 \mu$, and a Ge bolometer(Infrared Laboratories, Tucson, Arizona) with an NEP of $1 \times 10^{-13} \mathrm{~W} \mathrm{~Hz}^{-1 / 2}$ in the range 1 to $14 \mu$. It should be pointed out that detectors approximately 10 times more sensitive are currently available. 


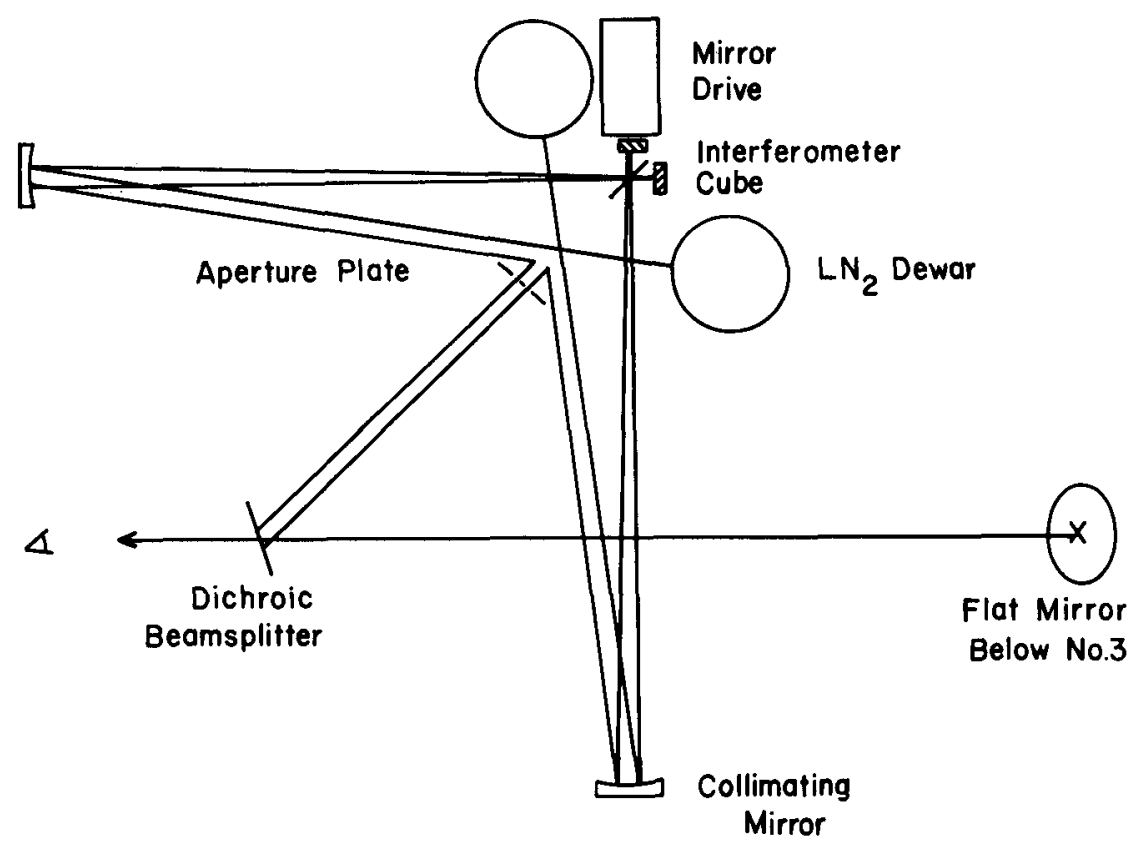

Fig. 1. Two-beam input foreoptics scheme for the FTS.

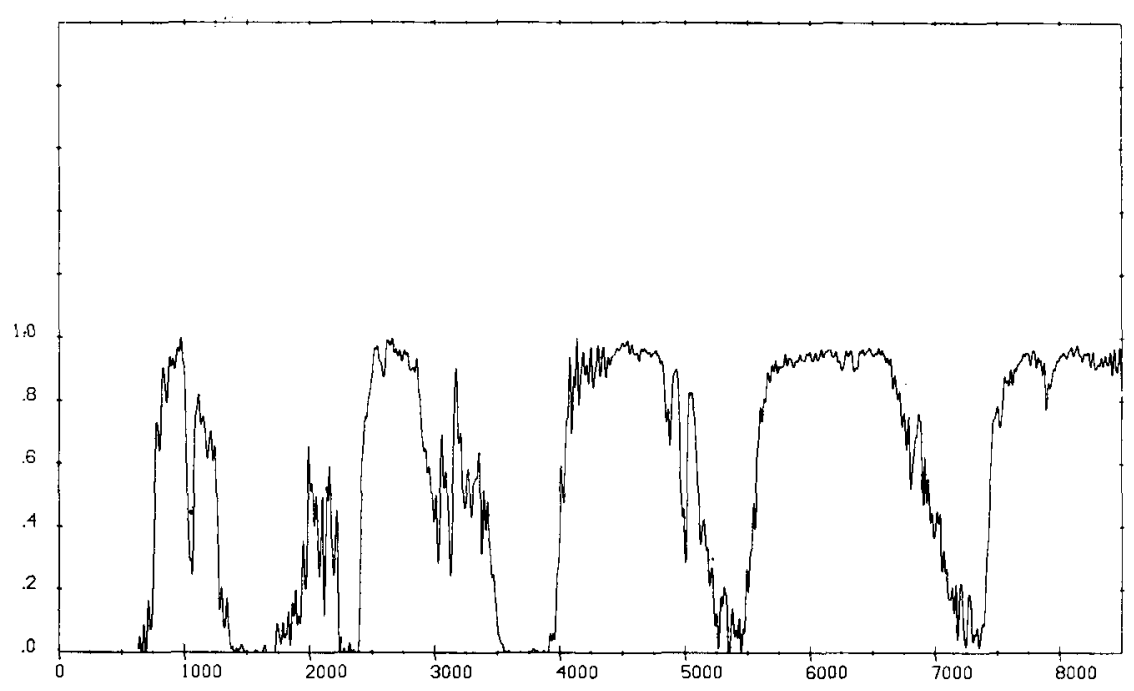

Fig. 2. Atmospheric transmission from Kitt Peak.

Figure 2 shows the atmospheric transmission in the range 700 to $9000 \mathrm{~cm}^{-1}$ for a typical good infrared night at Kitt Peak. Although the spectrometer has produced good spectra at up to $0.5 \mathrm{~cm}^{-1}$ resolution in each of these six atmospheric windows, I will restrict my discussion to the low resolution spectrophotometry.

The major non-instrumental difficulty to be surmounted was the development of 


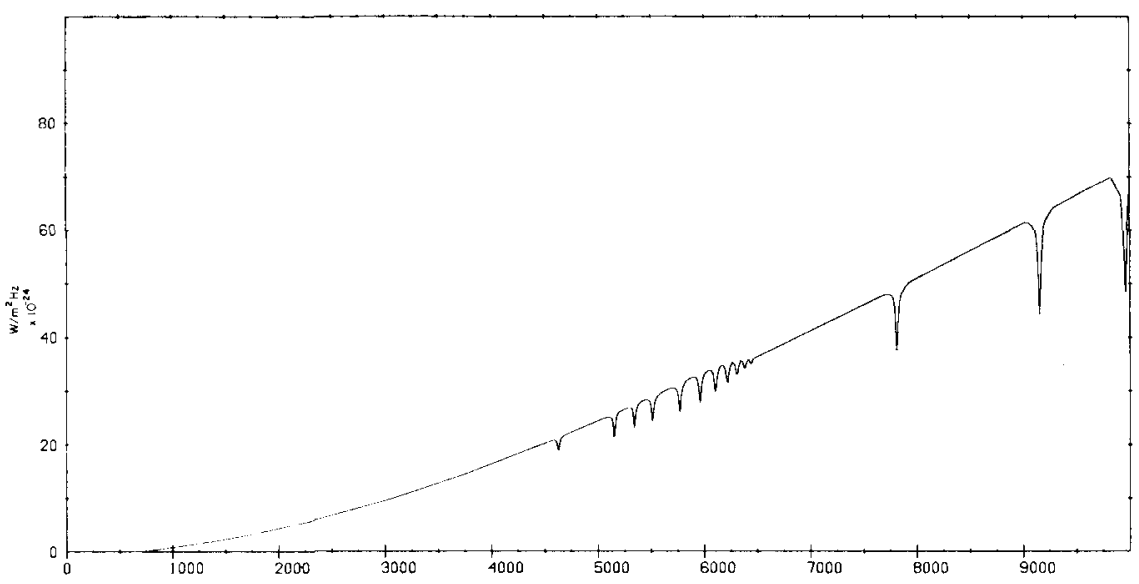

Fig. 3. Synthetic spectrum of Sirius,

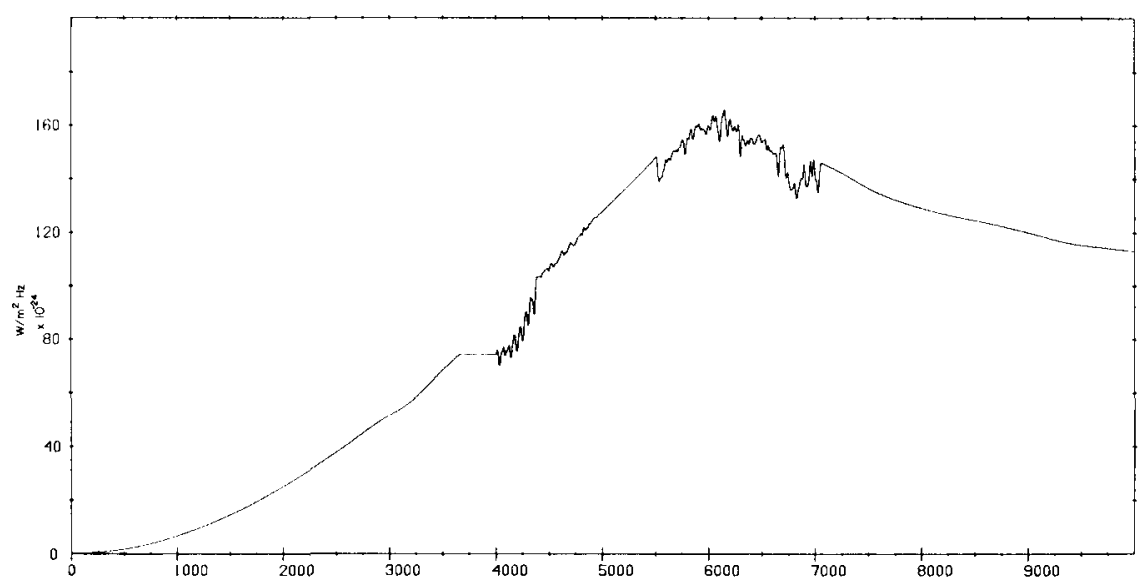

Fig. 4. Reference spectrum of Arcturus.

a suitable standard. In addition to having a known spectrum, the standard should have a high and reasonably uniform flux through the entire spectral region of interest. A computed synthetic spectrum of Sirius (Schild et al., 1971; Peterson, 1971) is shown in Figure 3. Throughout this spectral range the synthetic spectrum is completely consistent with observational data. Short of about $2500 \mathrm{~cm}^{-1}$ Sirius is too faint to serve as a spectrophotometric standard. In order to cover the full range, 700 to $10000 \mathrm{~cm}^{-1}$ a kind of hybrid spectrum was devised for the bright K-giant Arcturus.

Sirius was used to determine atmospheric transmission, which in turn was used to obtain a corrected spectrum of Arcturus in the region 4000 to $6500 \mathrm{~cm}^{-1}$. This was combined with the continuum flux predicted by model atmosphere studies, as shown in Figure 4. The spectral range 7000 to $10000 \mathrm{~cm}^{-1}$ has yet to be fitted with an observed spectrum. Also, the CO fundamental at $2000 \mathrm{~cm}^{-1}$ has not yet been taken into account. When this procedure has been completed, Arcturus will serve as a spectropho- 


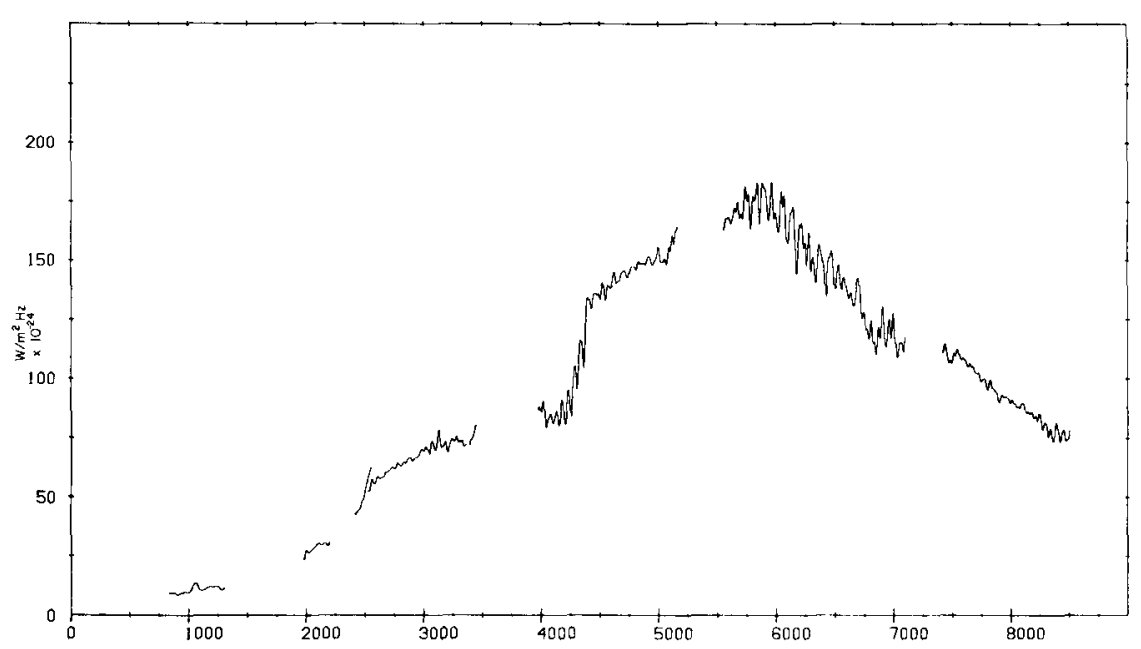

Fig. 5. The spectrum of $\alpha$ Her.

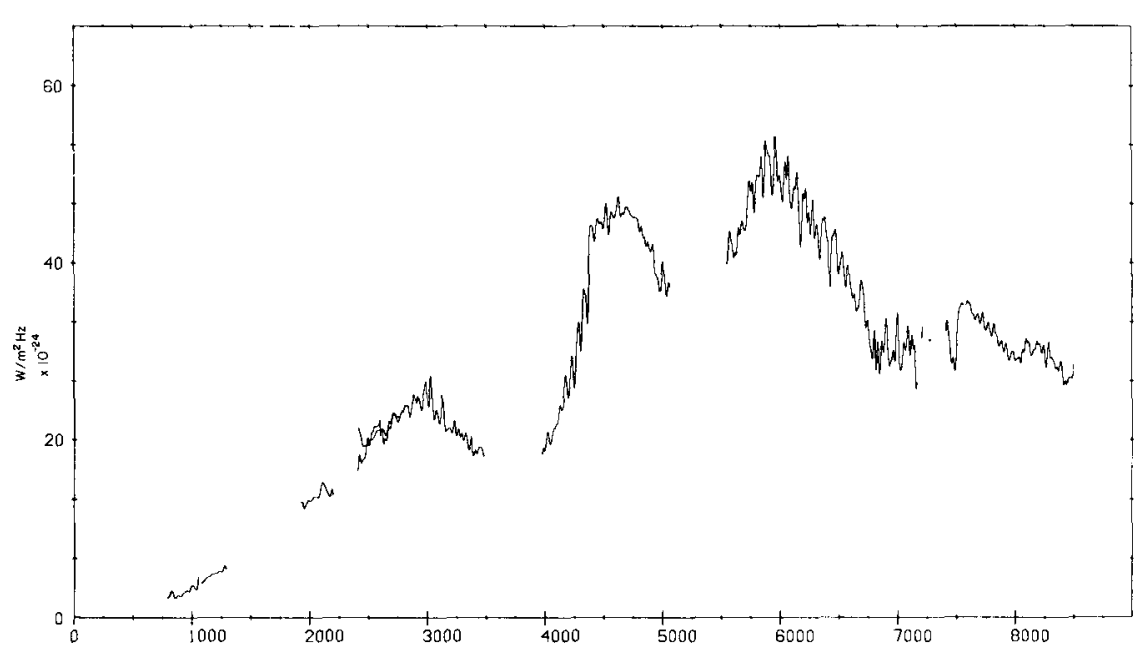

Fig. 6. The spectrum of RX Boo.

tometric standard over this very broad spectral range, ideal for reduction of the type of data described below.

In Figure 5, the spectrum of $\alpha$ Her from 800 to $8500 \mathrm{~cm}^{-1}$ is shown. This spectrum has been corrected for instrumental response and for telluric absorption using the Arcturus spectrum in its present form as described above. The range 800 to $3000 \mathrm{~cm}^{-1}$ was covered with a $\mathrm{Ge}$ on $\mathrm{KBr}$ beamsplitter, and the range 2500 to $8500 \mathrm{~cm}^{-1}$ with a $\mathrm{Si}$ on $\mathrm{CaF}_{2}$ beamsplitter. The absolute flux scale is established by reference to broad band filter photometry at $4500 \mathrm{~cm}^{-1}$. Throughout the rest of the spectrum fluxes are consistent with broadband photometry to within about $0.1 \mathrm{mag}$. 
The spectrum of $\alpha \mathrm{Her}$ is dominated by $\mathrm{CO}$ bands, atomic lines, and the $\mathrm{H}^{-}$ opacity minimum. Compare $3000 \mathrm{~K} \propto \mathrm{Her}$ with $2500 \mathrm{~K} \mathrm{RX}$ Boo in Figure 6. The luminosity classes are probably comparable, but the $500 \mathrm{~K}$ temperature difference leads to the sudden appearance of stellar water vapor absorption.

This very broad spectral bandwidth observational technique has been applied primarily to planets, and these examples of stellar spectra are merely illustrative. There are many potential applications, such as evaluation of blanketed model atmospheres (Querci et al., 1973) and determination of effective temperatures (Dyck et al., 1973).

\section{The K-Giant Survey}

I will now turn to a specific application of Fourier Transform Spectrophotometry - a study of $\mathrm{K}$ giants. The $\mathrm{K}$ giants are an especially fruitful subject for the infrared spectroscopist since they are relatively bright in the infrared, yet not so cool as to present the special complexities of polyatomic molecular formation. Also, an understanding of their composition will yield valuable information about the evolution of a star away from the main sequence.

Figure 7 shows the survey spectra of the six giants selected as normal standards with a comparison solar spectrum above. This spectral region is crowded with telluric absorption features, but these have been completely eliminated in the reduction

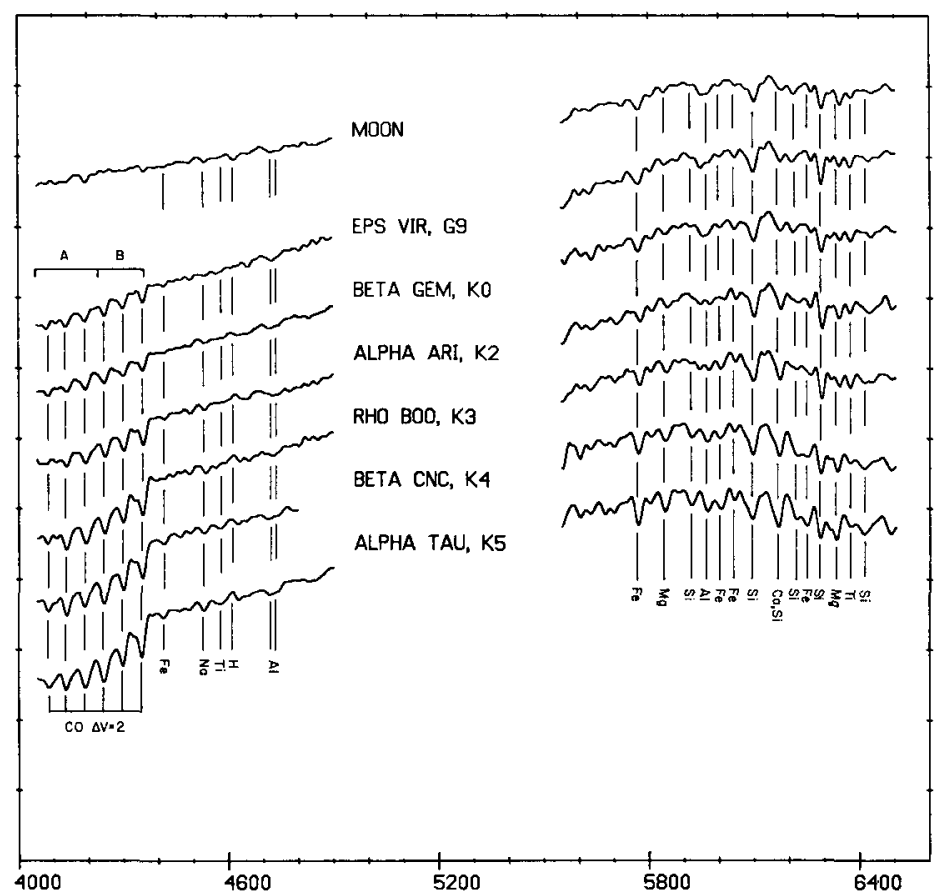

Fig. 7. $\mathrm{K}$ giants, $16 \mathrm{~cm}^{-1}$ resolution, normal standards. 
(Ridgway, 1972). The region 4900 to $5500 \mathrm{~cm}^{-1}$ is not plotted because of especially serious obscuration due to $\mathrm{CO}_{2}$ and $\mathrm{H}_{2} \mathrm{O}$.

Instrumental response has been removed from the spectra using nightly photometric calibration. As a check of photometric quality, the infrared index $H-K$ can be computed from these spectra and shows better than $1 \%$ agreement with infrared photometry. The standard deviation computed point by point is $\frac{1}{2} \%$ or better, except near the edges of the atmospheric windows where the noise is up to 3 times greater. All of the spectra are normalized in the same way, then shifted vertically by increments of one unit for ease of comparison. The zero flux level of the lowest spectrum coincides with the base of the plot.

At this resolution all of the spectral lines are blended, but as a guide the strongest contributors to some of the atomic features are indicated (Montgomery et al., 1969). The first overtone of $\mathrm{CO}$ is prominent starting with the $2-0$ bandhead at $4360 \mathrm{~cm}^{-1}$. The second overtone cannot be identified at this resolution. The general strengthening of features with later spectral type is due to increased abundances of neutral species and decreasing continuous opacity. Note the shift of the flux distribution to smaller wavenumber for the cooler stars. The distortion of the continuum due to the $\mathrm{H}^{-}$ opacity minimum at $6000 \mathrm{~cm}^{-1}$ is also prominent.

Survey spectra of four stars identified as SMR (Spinrad and Taylor, 1969) are shown in Figure 8. These stars are also known to have unusually strong $\mathrm{CN}$ bands (McClure, 1970). Figure 9 includes the rest of the survey spectra. This is a mixed group in-

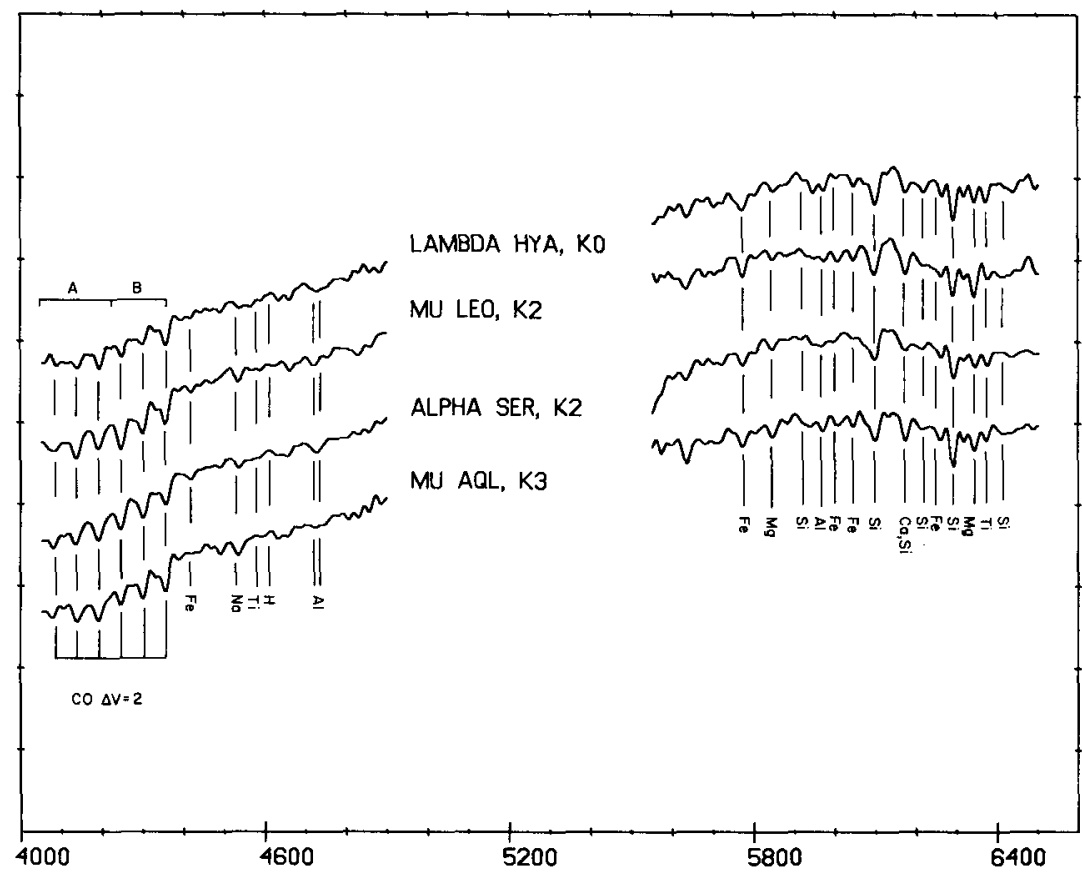

Fig. 8. K giants, $16 \mathrm{~cm}^{-1}$ resolution, super metal rich. 


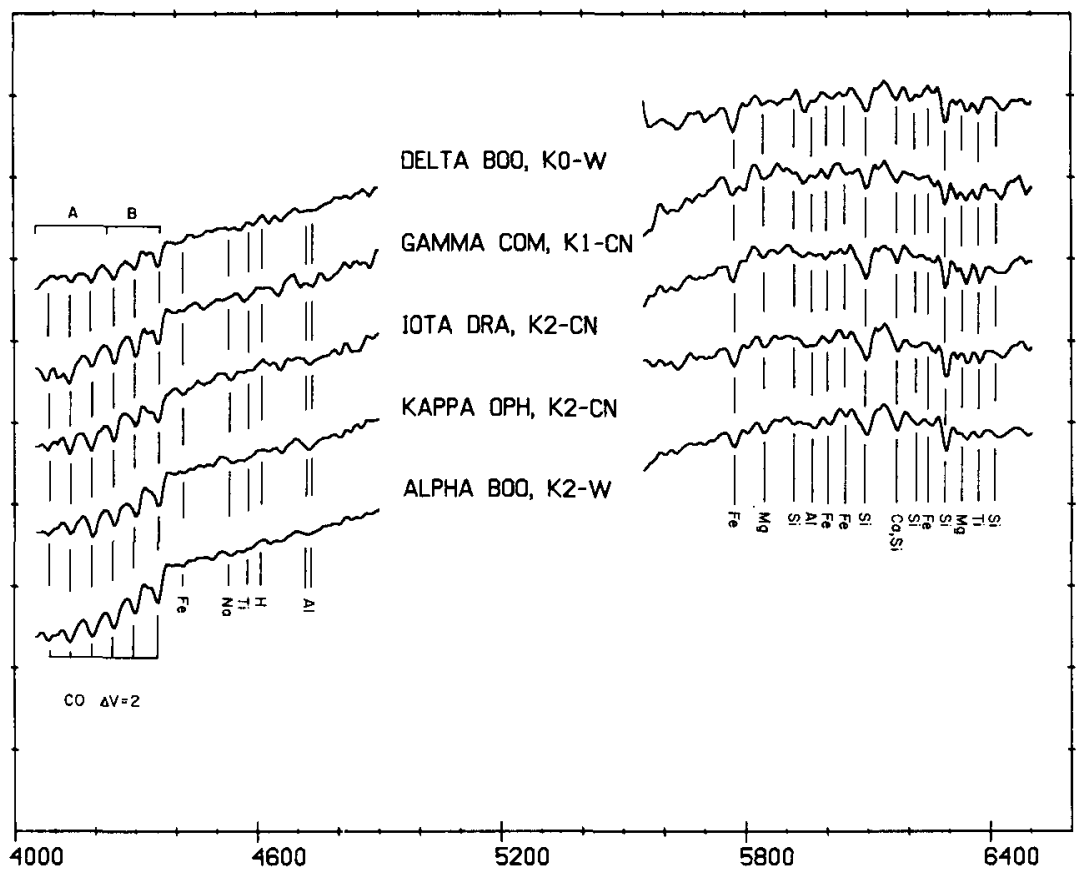

Fig. 9. K giants, $16 \mathrm{~cm}^{-1}$ resolution, $\mathrm{CN}$ strong and weak lined.

cluding CN strong stars which are not presently considered SMR, and two weak lined stars.

Because of the high photometric quality it is possible to mathematically 'synthesize' narrow and medium bandwidth filters to explore small differences among the spectra. As a first example, it is possible to define a temperature dependent color in the vicinity of the $\mathrm{H}^{-}$opacity minimum. This index can be compared with an index $T$ measured by Spinrad and Taylor near $7000 \AA$. Since the continuum opacity at $7000 \AA$ is much greater than at the minimum, the relationship between the two indices should be sensitive to stellar atmospheric temperature structure differences.

In Figure 10, $F(5875) / F(4525)$ is plotted against $T$ for the $\mathrm{K}$ giants studied. The dashed line through the normal stars represents the behavior expected from model computations. There is a clear dispersion in the sense that metal poor stars appear to have a relatively steeper temperature gradient with optical depth and the $\mathrm{CN}$ strong stars a relatively shallower temperature gradient. This is precisely opposite the effect we would expect if high metallicity and $\mathrm{CN}$ strength are associated with line blanketing. Differential blanketing in the filters might overwhelm any temperature structure effects but there is no evidence that this is the case here. Any other pair of filters in the range 4400 to $6800 \mathrm{~cm}^{-1}$ leads to a similar result.

By employing this filter 'synthesis' technique, it is possible to investigate the $\mathrm{CO}$ band strength in some detail. For the purpose of distinguishing the causitive mechanisms, we can study separately the integrated $\mathrm{CO}$ absorption for two regions. Region $\mathrm{A}$, 


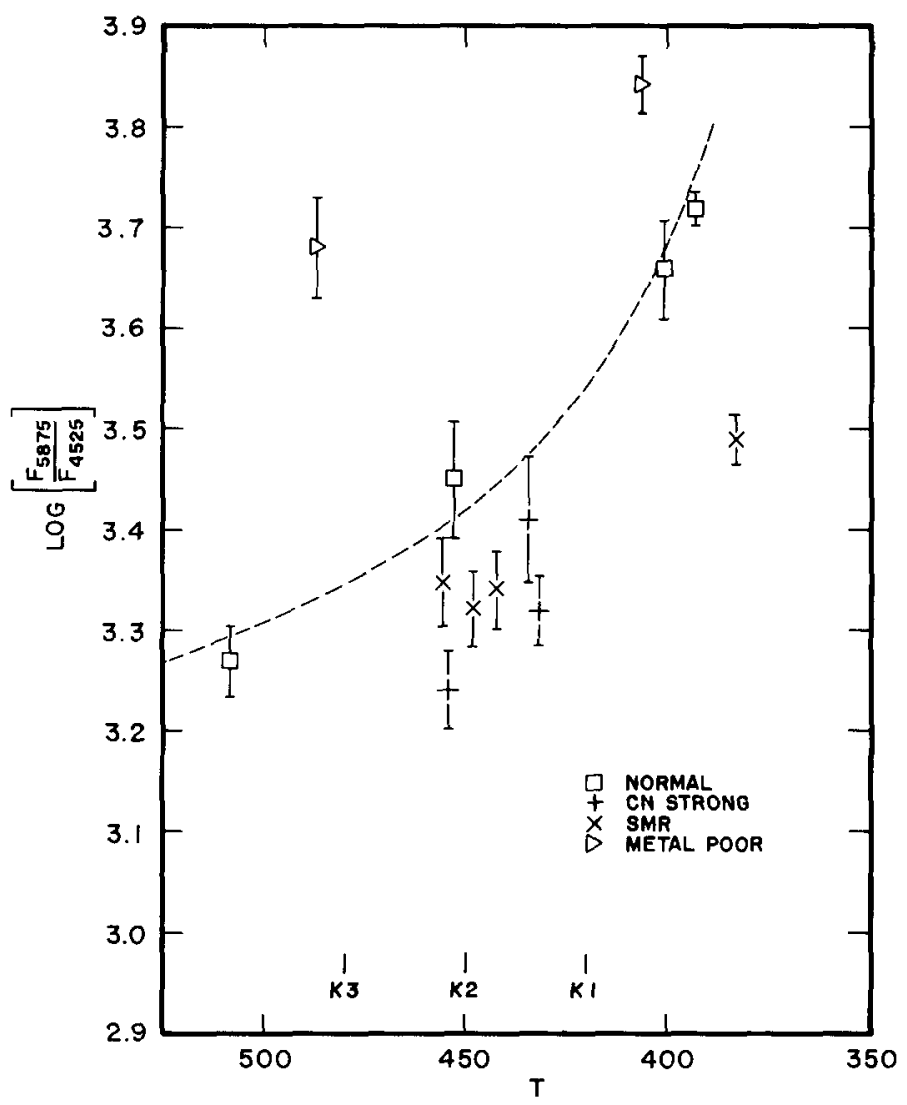

Fig. 10. $\log [F(5875) / F(4525)]$ vs $T$.

4000 to $4230 \mathrm{~cm}^{-1}$, is dominated by lines on the linear part of the curve of growth for giants earlier than about K4. Region B, 4230 to $4360 \mathrm{~cm}^{-1}$, is dominated by a relatively small number of very strong lines which are saturated for giants later than about $\mathrm{K} 0$.

A photometric study of CO band strength by Baldwin et al. (1973) employed a filter corresponding roughly to the region B. Since several stars were common to both studies, measures for these stars can be compared. In Figure 11 corresponding indices are plotted. The agreement is generally consistent with assigned observational error. The slope of the plotted points is consistent with the fact that Baldwin et al. used an adjacent rather than a local continuum.

Careful study of the spectroscopic data of this survey, and of the more extensive photometric survey of Baldwin et al. fails to reveal any systematic peculiarities in the $\mathrm{CO}$ band strength of the saturated region $\mathrm{B}$ for weak lined or $\mathrm{CN}$ strong stars.

Turning now to the unsaturated section of the $\mathrm{CO}$ bands, the integrated blocking fraction of region $\mathrm{A}$ is plotted against the temperature index $T$ in Figure 12. The smooth line is a theoretically predicted curve due to Schadee (1968) fitted to the normal $\mathrm{K}$-giants. Clearly all $\mathrm{CN}$ strong and SMR stars show excessive CO strengths in region 


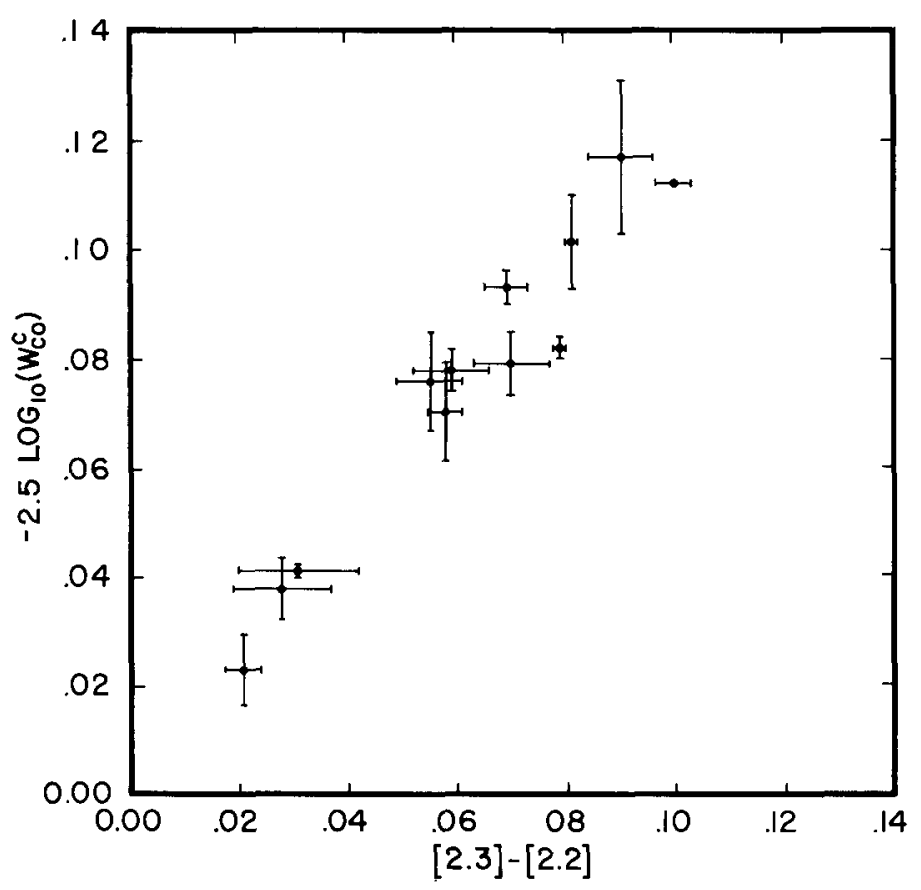

Fig. 11. $W_{\mathrm{Co}^{\mathrm{B}}}$ vs $(2.3)-(2.2)$.

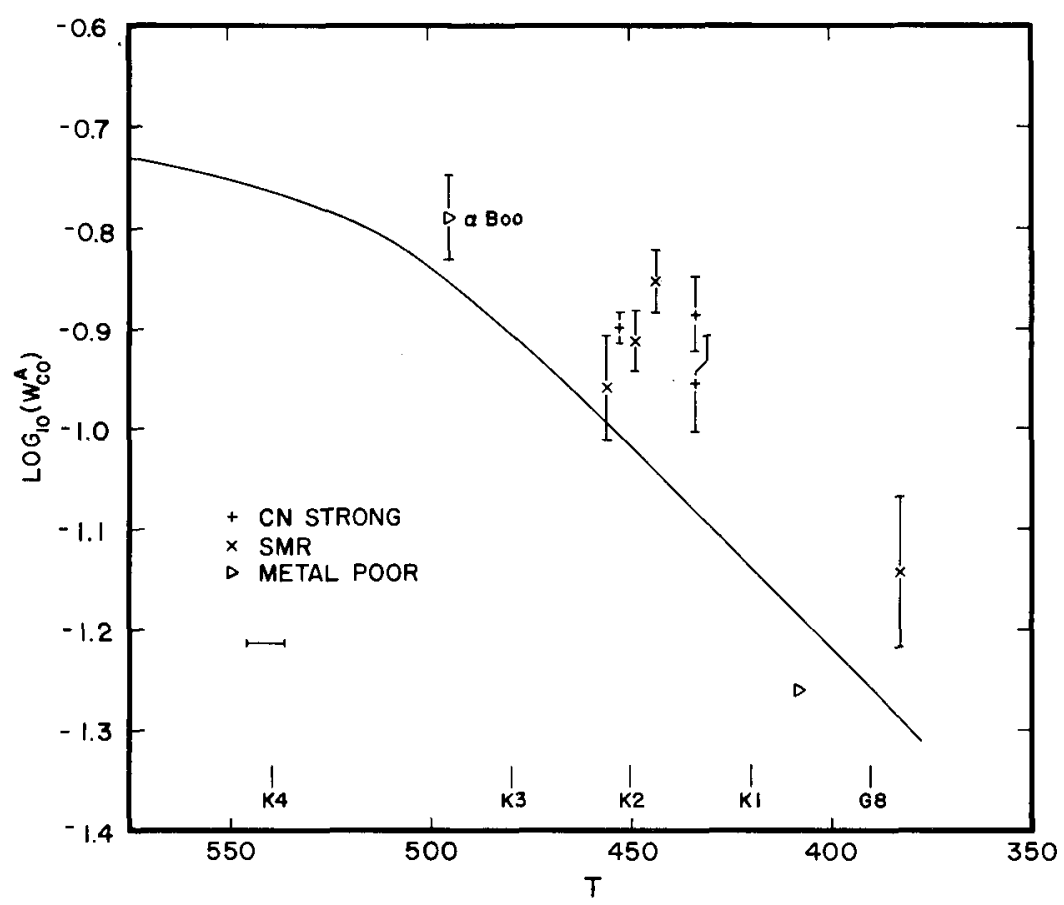

Fig. 12. $W_{\mathrm{CO}^{\mathrm{A}}}$ vs $T$. 
A. This might be due to differences in luminosity, microturbulent velocity, line blanketing, systematic errors in the $T$ index, or abundance differences.

On the basis of Schadee's work and the surface gravities tabulated by Williams $(1971,1972)$, it can be verified that the results of Figure 12 are unaffected by luminosity differences, except for $\alpha$ Boo which appears above the normal curve due to its substantially greater luminosity.

If the $\mathrm{CO}$ excesses were due to differences in microturbulent velocity, line blanketing with consequent surface cooling, or systematic errors in the $T$ index, we would expect saturated region $\mathrm{B}$ of the $\mathrm{CO}$ bands to exhibit an equal or greater variation in $\mathrm{CO}$ strength. In fact, as noted above, it does not. This is consistent with a genuine abundance difference, since saturated region $B$ would be less sensitive to abundance differences than unsaturated region $\mathrm{A}$. By studying the details of the correlation between $\mathrm{CO}$ and $\mathrm{CN}$ strengths, it may be possible to isolate the abundance peculiarities responsible.

\section{The ${ }^{12} \mathrm{C} /{ }^{13} \mathrm{C}$ Ratio}

As a final topic I would like to describe briefly an application of spectral synthesis techniques to the evaluation of the ${ }^{12} \mathrm{C} /{ }^{13} \mathrm{C}$ ratio from medium resolution spectra. The synthesis method was implemented by Carbon (1972), based on the model stellar atmosphere program SOURCE (Gingerich and Carbon, 1973). From a model atmosphere and a line list, the program solves the equations of state (under the assumption of LTE) at each specified depth to determine the molecular number densities. Then for each wavelength the source function is computed at each depth. The source function is then integrated over depth to find the emergent flux. The line absorption profile is assumed to be described by a Voigt function.

Of course, the greatest potential of this technique will be realized in the study of the highest resolution spectra; but it is also the best approach to the analysis of medium resolution spectra such as those in Figures 13 and 14 . Here, at $4 \mathrm{~cm}^{-1}$ resolution, the ${ }^{13} \mathrm{C}^{16} \mathrm{O}$ bandheads are clearly resolved, yet each resolution element contains many unresolved lines.

As a guide to the identification of atomic features, the positions of strong lines in $\alpha$ Boo (Montgomery et al., 1969) are indicated. Resolution was not sufficient to identify many comparably strong lines in both earlier and later spectral types. The CO $\Delta v=3$ sequence, although labeled, is not clearly distinguishable. The study of a spectrum involves the selection of suitable effective temperature, surface gravity and composition; generation of a model atmosphere; selection of microturbulent velocity and ${ }^{12} \mathrm{C} /{ }^{13} \mathrm{C}$ ratio; and synthesis of an 'infinite' resolution spectrum. The synthetic spectrum is then convolved with an appropriate line profile and compared with the observed spectrum. Model parameters can then be adjusted to obtain a satisfactory fit. The technique has been applied to $0.5 \mathrm{~cm}^{-1}$ resolution carbon star spectra (Carbon et al., 1973) and will be applied shortly to similar spectra of a number of $\mathrm{K}$ and $\mathrm{M}$ giants. I have recently used this technique to obtain ${ }^{12} \mathrm{C} /{ }^{13} \mathrm{C}$ ratios for these spectra.

Note first that $\alpha$ Ser shows weak or absent ${ }^{13} \mathrm{C}^{16} \mathrm{O}$ bands at this resolution. 


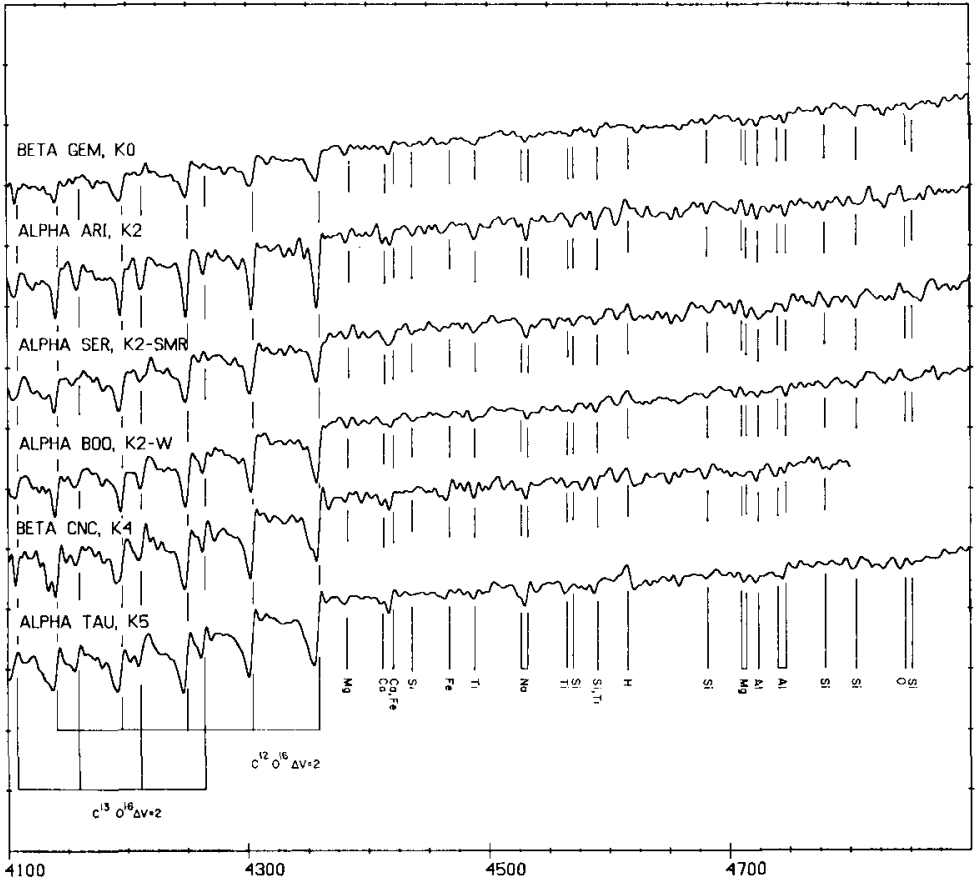

Fig. 13. K giants, $4 \mathrm{~cm}^{-1}$ resolution -4100 to $4900 \mathrm{~cm}^{-1}$.

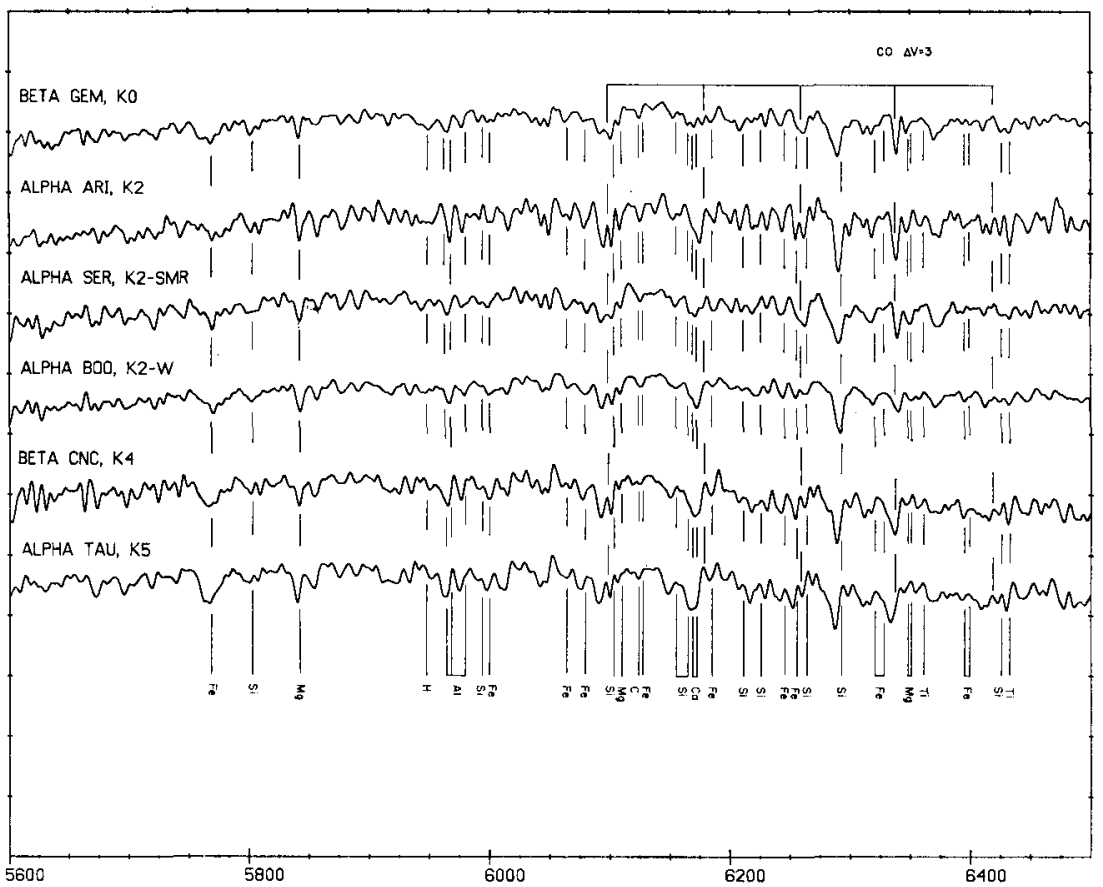

Fig. 14. K giants, $4 \mathrm{~cm}^{-1}$ resolution -5600 to $6500 \mathrm{~cm}^{-1}$. 


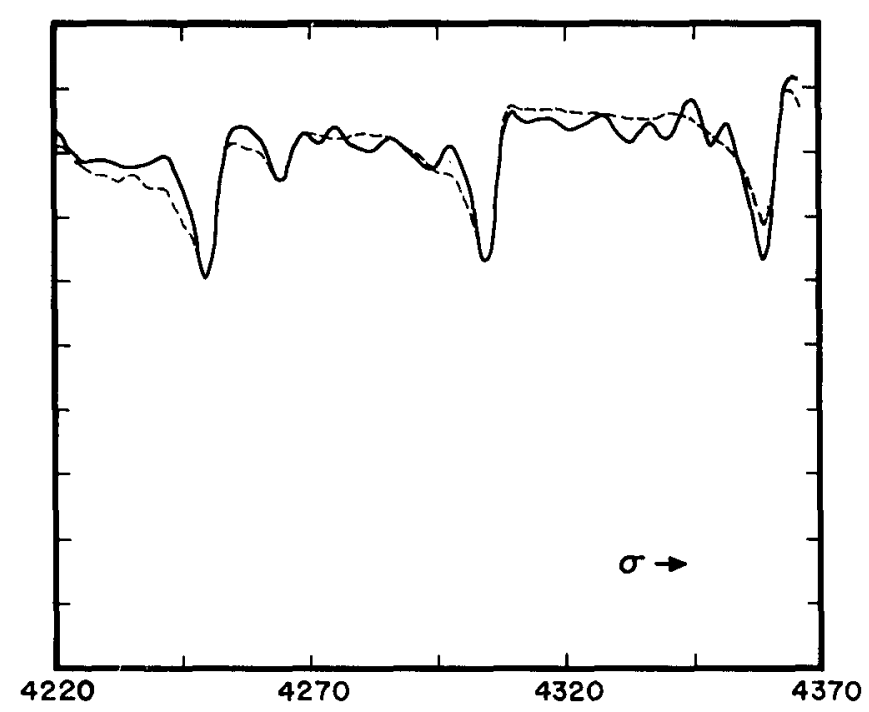

Fig. 15. $\alpha$ Ari observation, solid line, and synthetic spectrum, dashed line.

For the rest of the stars, $\mathrm{a}^{12} \mathrm{C} /{ }^{13} \mathrm{C}$ ratio of 10 yields a satisfactory fit to the observations. A typical example is shown in Figure 15 for $\alpha$ Ari. The dashed line is the synthetic spectrum. The fit is generally consistent with the $S / N$ of 100 and the presence of several strong atomic lines in the stellar spectrum.

$\mathrm{A}^{12} \mathrm{C} /{ }^{13} \mathrm{C}$ ratio of 10 is roughly consistent with several studies of $\alpha$ Boo especially by Lambert and Dearborn (1972) at higher resolution and with other molecules. Since evidence now indicates that a terrestrial ${ }^{12} \mathrm{C} /{ }^{13} \mathrm{C}$ ratio is probably typical throughout the solar system and the local interstellar medium (Ridgway, 1972), it is reasonably certain that these stars have substantial ${ }^{13} \mathrm{C}$ enhancement over the primordial abundances.

\section{References}

Baldwin, J. R., Frogel, J. A., and Persson, S. E.: 1973, Astrophys. J. 184, 427.

Carbon, D. F.: 1972, unpublished Ph.D. dissertation, Harvard University.

Carbon, D. F., Thompson, R. I., and Ridgway, S. T.: 1973, in preparation.

Dyck, H. M., Lockwood, G. W., and Capps, R. W.: 1973, to be published.

Gingerich, O. and Carbon, D. F.: 1973, Smithsonian Observatory Special Report No. 300, in preparation.

Lambert, D. L. and Dearborn, D. S.: 1972, Mem. Soc. Roy. Sci. Liège, 6th Ser. 3, 147.

McClure, R. D.: 1970, Astron. J. 75, 41.

Montgomery, E. F., Connes, P., Connes, J., and Edmonds, F. N.: 1969, Astrophys. J. Suppl. 19, 1. Peterson, D. M.: 1971, private communication.

Querci, F., Querci, M., and Tsuji, T.: 1973, to be published.

Ridgway, S. T.: 1972, Ph.D. dissertation, SUNY at Stony Brook, University Microfilms, Ann Arbor, Michigan.

Schadee, A.: 1968, Astrophys. J.: 151, 239.

Schild, R., Peterson, D. M., and Oke, J. B.: 1971, Astrophys. J. 166, 95.

Spinrad, H. and Taylor, B. J.: 1969, Astrophys. J. 157, 1279.

Williams, P. M.: 1971, Monthly Notices Roy. Astron. Soc. 153, 171.

Williams, P. M.: 1972, Monthly Notices Roy. Astron. Soc. 158, 361. 


\section{DISCUSSION}

Townes: You have given a ratio of 10 for ${ }^{12} \mathrm{C}$ and ${ }^{13} \mathrm{C}$ and $\mathrm{I}$ wonder if you could say what the general range of variability would allow in your analysis?

Ridgway: The uncertainties are mostly hidden in the sense that they are not observational errors. My value of 10 compared with Lambert's results of 6 or 7 certainly is not inconsistent in any way. 5 to 20 would be fair enough.

Williams: I am most interested in your comparison of your carbon monoxide excess with your cyanogen excess of your super metal rich and cyanogen strong stars. The error bars were large, but there did seem to be a slight systematic difference between them, in the sense that the super metal rich stars were of a slightly stronger cyanogen for their carbon monoxide. This suggests that whereas the strong $\mathrm{CN}$ stars may be mostly carbon strong, the super metal rich stars are also nitrogen strong. And I think you will see $\mathrm{CO}$ photometry seems to be an extremely valuable way of finding out just what is happening among these stars. 